This is the submitted version of the article:

López-Suárez, M.; Pruneda, M.; Abadal, G.; Rurali, R..

Piezoelectric monolayers as nonlinear energy harvesters.

Nanotechnology, (2014). . : - .

10.1088/0957-4484/25/17/175401.

Available at:

https://dx.doi.org/10.1088/0957-4484/25/17/175401 


\title{
Piezoelectric monolayers as non-linear energy harvesters
}

\section{Miquel López-Suárez}

Departament d'Enginyeria Electrònica; Universitat Autònoma de Barcelona; 08193

Bellaterra, Barcelona, Spain

\section{Miguel Pruneda}

ICN2-Institut Català de Nanociència i Nanotecnologia, Campus UAB, 08193

Bellaterra, Barcelona, Spain

CSIC-Consejo Superior de Investigaciones Científicas, ICN2 Building, Campus UAB, 08193 Bellaterra, Barcelona, Spain

\section{Gabriel Abadal}

Departament d'Enginyeria Electrònica; Universitat Autònoma de Barcelona; 08193

Bellaterra, Barcelona, Spain

\section{Riccardo Rurali}

E-mail: rrurali@icmab.es

Institut de Ciència de Materials de Barcelona (ICMAB-CSIC), Campus de Bellterra, 08193 Bellaterra, Barcelona, Spain

\begin{abstract}
We study the dynamics of h-BN monolayers by first performing $a b$ initio calculations of the deformation potential energy and then solving numerically a Langevine type equation to explore their use in non-linear vibration energy harvesting devices. An applied compressive strain is used to drive the system into a non-linear bistable regime, where quasi-harmonic vibrations are combined with low frequency swings between the minima of a double-well potential. Due to its intrinsic piezoelectric response, the non-linear mechanical harvester naturally provides an electrical power that is readily available or can be stored by simply contacting the monolayer at its ends. Engineering the induced non-linearity, a $20 \mathrm{~nm}^{2}$ device is predicted to harvest an electrical power of up to $0.18 \mathrm{pW}$ for a noisy vibration of $5 \mathrm{pN}$.
\end{abstract}


Two-dimensional materials, such as graphene and other monolayer systems [1] exhibit many properties that render them promising candidates for electromechanical actuation [2]. Suspended nanostructures that combine a low mass density and relatively large areas seem to be ideally suited for the harvesting of mechanical vibrations and other energy sources that are freely available [3,4]. Harmonic oscillators are, in most of the cases, very good approximations of the dynamical behavior of these systems. Nevertheless, a harmonic oscillator is very sensitive around a set of frequencies -its resonant frequencies- but responds poorly otherwise. Unfortunately, ambient vibrations and thermo-mechanical noise have normally a very broad frequency spectrum that extends into the very low frequency region (typically below $100 \mathrm{~Hz}$ ). Harmonic oscillators that respond in such frequency range, besides being intrinsically inefficient, would be extremely large, making their use in self-powering of small devices impractical. To overcome this limitation, mechanical harvesters with non-linear potentials have been analyzed and compared to linear resonators [5] as optimal alternatives when the energy sources are stochastic and characterized by a wideband spectrum [6-8].

Following the ground-breaking work of Cottone et al. [9], in a previous study we have shown how engineered non-linearities can increase the efficiency of ambient vibration harvesting of a suspended graphene nanoribbon [10]. By compressively straining it, the graphene sheet is driven into a bistable regime that maximizes the root mean square (rms) of the displacement, thus the mechanical power. However, harvested mechanical power is difficult to use and it is desirable to convert it into electrical energy, which can be straightforwardly stored. Although piezoelectricity -the electrical response to applied mechanical stress- is not one of the many outstanding properties of graphene, non-centrosymmetric monolayers have been predicted to have remarkable intrinsic piezoelectric coefficients [11].

In this work we discuss the nanoscale implementation of a bistable device based on piezoelectric h-BN capable of harvesting thermo-mechanical noise and low frequency vibrations from the environment, yielding an electrical voltage due to its intrinsic electromechanical response. Remarkably, the generated voltage is mostly independent of the degree of strain, as long as the resonator operates in the bistable regime.

Our device consists of a $20 \mathrm{~nm}$ long (17 nm suspended) single-layer h-BN subjected to compressive strains ranging from 0 to $2 \%$. Upon compression the monolayer favors two buckled ground states, symmetric with respect to the plain defined by the ideal flat layer. A sketch of the system is shown in Fig. 1. As in our previous work [10], we calculated the deformation potential of the vibrating system, and the changes in induced polarization due to the mechanical deformation by means of atomistic firstprinciples calculations within density-functional theory (DFT) as implemented in the SIESTA package [12]. Similarly to our previous work [10], for each value of the strain we generate several sinusoidal deformations with an increasing amplitude and we calculate the total energy and the polarization for each of these configurations.

To reduce the computational load, we consider the two-dimensional material, rather than a nanoribbon with finite width. The energies quoted in the text have been obtained 
by rescaling the results obtained for the computational cell and thus neglect edge effects, which can generate stress fields and ripples. These effects are not accounted for in our model. However, the dynamic response of the system is not expected to qualitatively change.

Changes in polarization are calculated using the standard Berry's phase approach [13]. The Brillouin zone was sampled with a grid of $1 \times 14 \times 1$ k-points. We used norm-conserving pseudopotentials to account for the core electrons and the generalized gradient approximation (GGA) for the exchange-correlation functional [14]. We used a carefully optimized single- $\zeta$ polarized basis set to expand the one-electron wave function [15]. This lighter basis set allowed us to deal efficiently with the large number of structures involved and with the computationally intensive calculations of the polarization, maintaining a very good accuracy (tests against a high-quality optimized double- $\zeta$ basis set gave lattice parameter and piezoelectric constant of h-BN within 3 and $2 \%$, respectively).

The two minimum energy configurations that appear under compressive strain are separated by a barrier whose maximum corresponds to the strained, flat layer (see Fig. 2). Increasing the compression leads to higher barriers and larger separation between the minima, as shown in the left inset (see also Ref. [10]). Notice that $E_{s}(\epsilon)=\left.E(\epsilon)\right|_{z=0}-\left.E(0)\right|_{z=0}$ is the energy cost of sustaining a strain $\epsilon$. By performing a similar set of calculation for tensile strains the uniaxial Young modulus can be estimated as $Y=\left.\frac{1}{V_{0}} \frac{\partial^{2} E_{s}}{\partial \epsilon^{2}}\right|_{\epsilon=0}$, where $V_{0}$ is the equilibrium volume [16] (right inset of Fig. 2). We obtain a value of $0.75 \mathrm{TPa}$, in good agreement with previous results [17].

Non-centrosymmetric planar BN sheet develops an electric dipole moment either by (i) the applied uniaxial strain (direct piezoelectric effect, $z=0$ and $\epsilon>0$ ), (ii) the elastic deformation induced by the vibrating mode (flexoelectric effect, $z \neq 0$ and $\epsilon=0$ ) or (iii) a combination of the two $(z \neq 0$ and $\epsilon>0)$. The uniaxial strain is the simplest of these cases: it yields an electrical polarization aligned with the straining direction which depends linearly on the strain through the piezoelectric constant of the material, $P=\alpha \epsilon$. We have estimated this parameter by applying increasingly higher uniaxial strains to the BN sheet and calculating the polarization, obtaining a value of $3.085 \cdot 10^{-10} \mathrm{C} / \mathrm{m}$ in good agreement with previously published results [18]. The situation becomes considerably more intricate when out of plane displacements are involved. As discussed in detail by Naumov et al. [18], these configurations feature not only a finite strain, but also a strain gradient. Therefore the polarization also includes a flexoelectric term, beside the conventional piezoelectric response. Our numerical results are in excellent agreement with the phenomenological expression of $P$ induced by a sinusoidal out-of-plane displacement of Ref. [18] (circles and continuous line in the inset of Fig. 3, respectively). The calculated values of the polarization as a function of the applied strain and of the amplitude of the sinusoidal vibration (symbols in Fig. 3) can be satisfactorily fitted to a biquartic polynomial (continuous lines in Fig. 3).

We now move to the study of the dynamical behavior of the device, which is 
described by the equation of motion:

$$
m \ddot{z}=-\frac{\partial E}{\partial z}-b \dot{z}+F_{0} \xi(T)
$$

where $E$ is the elastic (potential) energy as obtained by the ab initio calculations and reported in Fig. 2, $m$ is the effective mass (taken to be 0.4 times the total mass [19]), and $b$ is a viscous damping coefficient [20] related to the quality factor $Q$ through $b=2 \pi f_{0} m / Q$. We have considered $Q=100$ throughout all this work [21,22]. $F_{0} \xi(T)$ represents a random force with flat spectrum that reproduces the main characteristics of most kinds of environmental vibrations. We take a noise intensity of $5 \mathrm{pN}$, which suits most vibrational ambient noise [23] (for comparison, $1 \mathrm{pN}$ corresponds to room temperature pure thermo-mechanical noise).

Notice that having fitted the calculated polarizations for arbitrary compressions, together with the generalized position $z$, we can also trace the dynamical evolution of the polarization and, consequently, of the generated voltage across a load $R_{L}$ by solving [24]

$$
\begin{aligned}
m \ddot{z} & =-\frac{\partial E}{\partial z}-b \dot{z}-\Gamma_{1} \frac{\partial P_{x}^{2 D}}{\partial z} \dot{z}+F_{0} \xi(T) \\
\dot{V} & =\Gamma_{2} \frac{\partial P_{x}^{2 D}}{\partial z} \dot{z}-\frac{1}{R_{L} C_{p}} V
\end{aligned}
$$

where $P_{x}^{2 D}$ is the $2 \mathrm{D}$ polarization along $x, C_{p}$ is the capacity of an infinite parallel wire capacitor analogous to our system [25], $\Gamma_{2}=w / C_{p}, w$ being the layer thickness, is the electromechanical coupling coefficient [26] and $\Gamma_{1}=\Gamma_{2} / C_{p} . R_{L}$ is taken to be $2.4 \mathrm{G} \Omega$, which is the impedance matched value that maximizes the electrical power (see below).

The device exhibit three distinct modes of operation that are summarized in Fig. 4, where we plot $z$ and $V$ as a function of time (left and right column, respectively). At zero or moderate compressive strains the BN sheet vibrate around the equilibrium position of the unstrained system, i.e. the flat sheet, and a voltage of at most $1 \mathrm{mV}$ is generated (bottom panels). As the strain increases the two potential wells appear: the system will vibrate around the equilibrium position of one of them, but if the barrier is not too high it will be able to stochastically swing to the other well. When bistability kicks in the greatly increased $z_{r m s}$ results in a voltage which is almost one order of magnitude larger (middle panels). Finally, for high values of the strain, the barrier will be high enough for the system to remain trapped in one of the two minima (top panels). Interestingly, although $z_{r m s}$ is reduced and is even smaller than for the uncompressed sheet, the generated voltage is of the same order of the purely bistable case (top panels).

To further investigate the evolution of the dynamics with stress, Fig. 5 shows the rms of the generalized coordinate $z$ and the harvested electrical power [9], $P_{r m s}=$ $V_{r m s}^{2} / R_{L}$, are plotted as a function of the applied strain. Increasing the strain drives progressively the vibrating sheet into the bistable regime, $z_{r m s}$ goes through a steep increase and the harvested power follows it. At $\epsilon \sim 0.3 z_{r m s}$ is maximum and features a sharp peak: slightly larger strains trap the system in one of the minima of the doublewell potential. In this regime, nonetheless, the harvested power starts to be dominated by the polarization, which experiences very large changes even for small variations of 
the amplitude of the vibrating mode. This increase of $\frac{\partial P_{x}^{2 D}}{\partial z}$ almost entirely balances the reduction in $z_{r m s}$. This fact has important consequences from the device design viewpoint, because one can pursue the optimal compression $\epsilon \sim 0.3$ without risking that small errors can suddenly drive the system into an operation regime where the efficiency is even lower than with the unstrained sheet. When the optimal strain is exceeded, the device progressively recovers a quasi-linear behavior with an harvested electrical power that is still within $85 \%$ its maximum value.

We conclude by observing that the overall performance in term of energy harvesting has two components: (i) the intrinsic properties of the material, such as the piezoelectric coefficient or the Young's modulus and (ii) the way the device is engineered. Although these two components are not independent (the optimal strain, for instance, depends on the material compressibility), it seems that other 2D materials with a larger piezoelectric response would in principle result in more efficient harvesting. A good candidate is $\mathrm{MoS}_{2}$, which has larger piezoelectric coefficients (in good agreement with previous studies [11], we have obtained values of $3.87 \cdot 10^{-10}$ and $3.81 \cdot 10^{-10} \mathrm{C} / \mathrm{m}$ in the relaxedion and clamped-ion frameworks, respectively, to be compared with $3.085 \cdot 10^{-10}$ and $2.855 \cdot 10^{-10} \mathrm{C} / \mathrm{m}$ for $\left.\mathrm{h}-\mathrm{BN}\right)$. Unfortunately, we could not observe buckling of the $\mathrm{MoS}_{2}$ sheet, as it is much stiffer than one atom thick h-BN concerning out-of-plane displacements. The larger piezoelectric coefficients do not compensate the lack of bistability and at this size the choice of h-BN still pays off. For longer ribbons, currently beyond our computational capabilities, buckling should be observed and the overall performances of a $\mathrm{MoS}_{2}$-based harvester could probably exceed those of h-BN. More work in this direction is needed.

In conclusions, we have shown that engineered non-linearities in piezoelectric twodimensional system such as monolayer h-BN can lead to sizeable energy harvesting of ambient vibrations, thermo-mechanical and other broad band energy source freely available. In particular, a $20 \mathrm{~nm} \times 1 \mathrm{~nm}$ h-BN monolayer under a compressive strain $\epsilon=0.3 \%$ can harvest up to $0.18 \mathrm{pW}$ from a $5 \mathrm{pN}$ vibration. Finally, the combination of piezoelectric and mechanical bistability properties of the suspended BN structures can pave the way for the future development of beyond CMOS devices that could perform sensing, computing and transmission functions at the nanoscale in a selfpowered way.

\section{Acknowledgments}

Funding from the Ministerio de Ciencia e Innovación under contract No ENE2009-14340CO2-02 (OPACMEMS) and FIS2012-37549, and from the EU-FP7 FET-Proactive Coordination Action, Project no. 270005 (ZEROPOWER), are greatly acknowledged. We thank the Centro de Supercomputación de Galicia (CESGA) for the use of their computational resources. 
Piezoelectric monolayers as non-linear energy harvesters

\section{References}

[1] Novoselov K S, Jiang D, Schedin F, Booth T J, Khotkevich V V, Morozov S V and Geim A K 2005 Proc. Natl. Acad. Sci. USA 102 10451-10453

[2] Huang Y, Liang J and Chen Y 2012 J. Mater. Chem. 223671

[3] Garcia-Sanchez D, van der Zande A M, San Paulo A, Lassagne B, McEuen P L and Bachtold A 2008 Nano Lett. 8 1399-1403

[4] van der Zande A M, Barton R A, Alden J S, Ruiz-Vargas C S, Whitney W S, Pham P H Q, Park J, Parpia J M, Craighead H G and McEuen P L 2010 Nano Lett. 10 4869-4873

[5] Méndez V, Campos D and Horsthemke W 2013 Phys. Rev. E 88(2) 022124

[6] Khovanova N A and Khovanov I A 2011 Appl. Phys. Lett. 99144101 (pages 3)

[7] Halvorsen E 2008 J. Microelectromech. Syst. 17 1061-1071

[8] Halvorsen E 2013 Phys. Rev. E 87(4) 042129

[9] Cottone F, Vocca H and Gammaitoni L 2009 Phys. Rev. Lett. 102080601

[10] López-Suárez M, Rurali R, Gammaitoni L and Abadal G 2011 Phys. Rev. B 84(16) 161401

[11] Duerloo K A N, Ong M T and Reed E J 2012 J. Phys. Chem. Lett. 3 2871-2876

[12] Soler J M, Artacho E, Gale J D, García A, Junquera J, Ordejón P and Sánchez-Portal D $2002 \mathrm{~J}$. Phys.: Condens. Matter 14 2745-2779

[13] King-Smith R D and Vanderbilt D 1993 Phys. Rev. B 47 1651-1654

[14] Perdew J P, Burke K and Ernzerhof M 1996 Phys. Rev. Lett. 77 3865-3868

[15] Anglada E, M Soler J, Junquera J and Artacho E 2002 Phys. Rev. B 66205101

[16] The equilibrium volume is defined by taking an effective thickness of $3.5 \AA$, which is the layer-tolayer separation in bulk h-BN.

[17] Peng Q, Ji W and De S 2012 Comput. Mater. Sci. 56 11-17

[18] Naumov I, Bratkovsky A M and Ranjan V 2009 Phys. Rev. Lett. 102(21) 217601

[19] Kaajakari V 2009 Practical MEMS (Las Vegas, Nevada: Small Grid Publishing)

[20] Atalaya J, Isacsson A and Kinaret J M 2008 Nano Lett. 8 4196-4200

[21] Bunch J S, van der Zande A M, Verbridge S S, Frank I W, Tanenbaum D M, Parpia J M, Craighead H G and McEuen P L 2007 Science 315 490-493

[22] Barton R A, Ilic B, van der Zande A M, Whitney W S, McEuen P L, Parpia J M and Craighead H G 2011 Nano Lett. 11 1232-1236

[23] López-Suárez M, Rurali R, Gammaitoni L and Abadal G 2013 Microelectronic Engineering 111 $122-125$

[24] Beeby S P, Tudor M J and White N M 2006 Measurement Science and Technology 17 R175-R195 ISSN 0957-0233

[25] A parallel wire capacitor has capacitance $C=\frac{\pi \epsilon w}{\operatorname{Ln}\left(l_{q} / r_{q}\right)}$, where $l_{q}$ is the separation between the charged edges, i.e. the length of the suspended ribbon, and $r_{q}$ is the effective radius of the edge states, which can be approximated to be one lattice parameter [18].

[26] Roundy S and Wright P K 2004 Smart Materials and Structures 13 1131-1142 


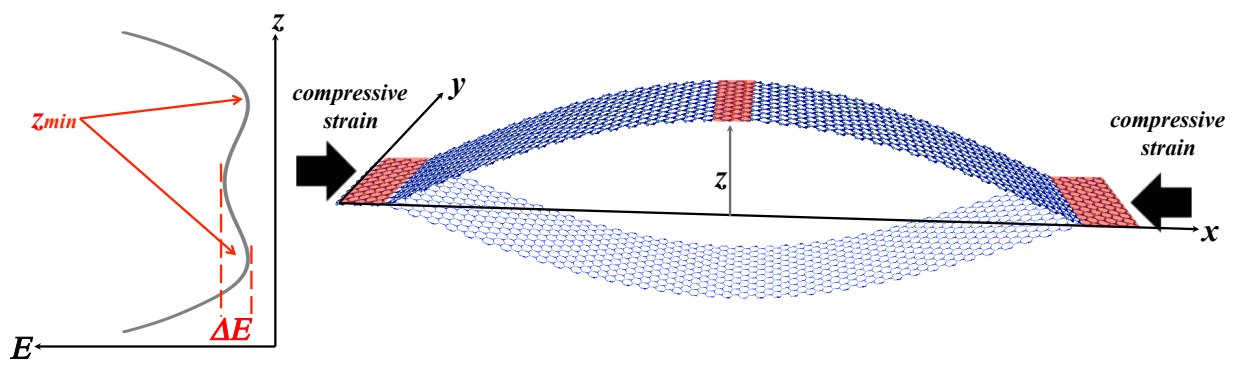

Figure 1. Sketch of the h-BN structure studied, highlighting the two symmetric equilibrium configurations under compressive strain. The double-well potential is shown on the left-hand side. 


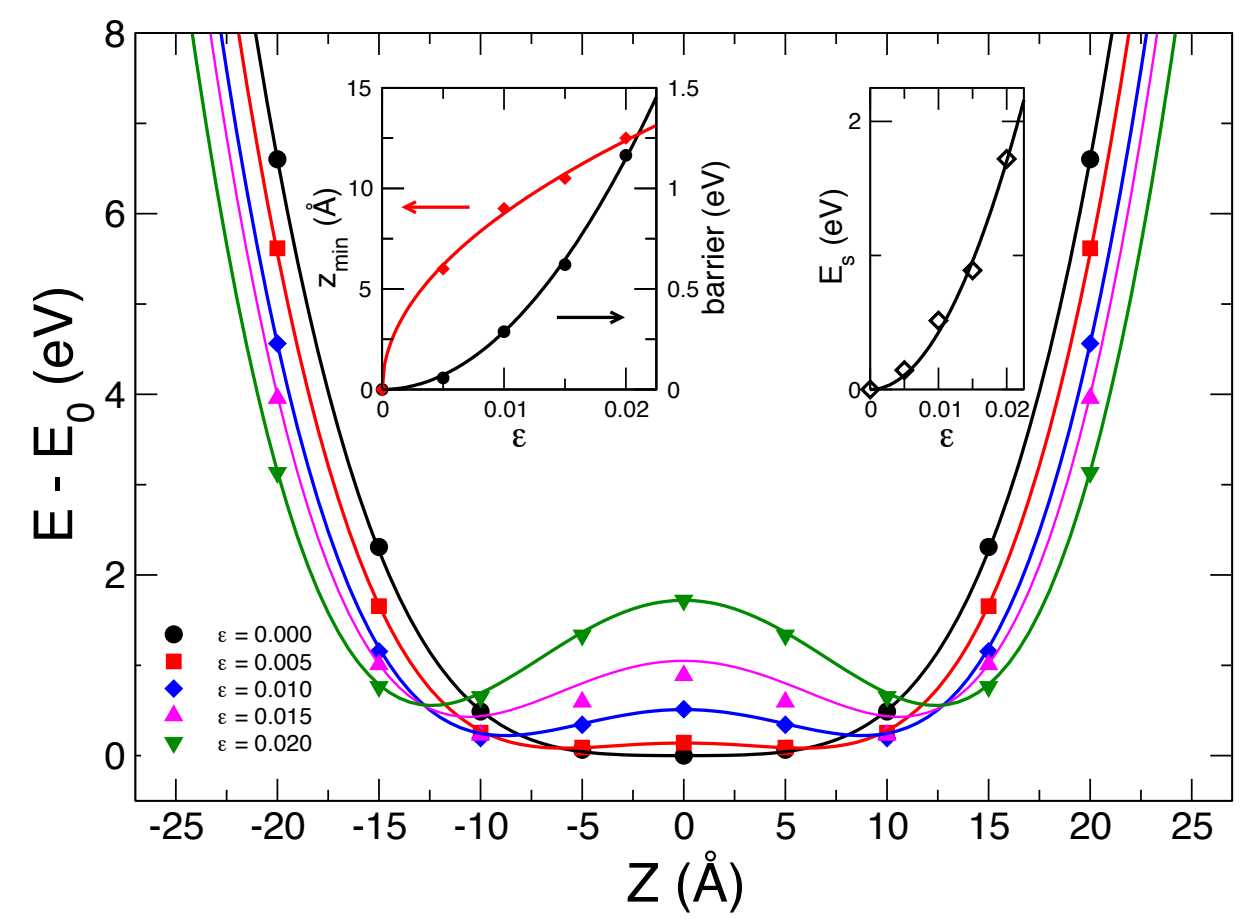

Figure 2. Potential energy as a function of the amplitude of the sinusoidal deformation for different values of the compressive strain $\epsilon$. The position of the minima and the height of the barrier between them are plotted in the left inset (red and black symbols, respectively). Fits (continuous line) reveal the $\sqrt{\epsilon}$ and $\epsilon^{2}$ dependence of these two quantities. Energies of the main panel are referred to the energy of the flat and unstrained h-BN sheet. The right inset show how the energy increases upon straining, i.e. the strain energy. Its dependence on the strain $\epsilon$ is used to extract the Young's modulus (see text). 


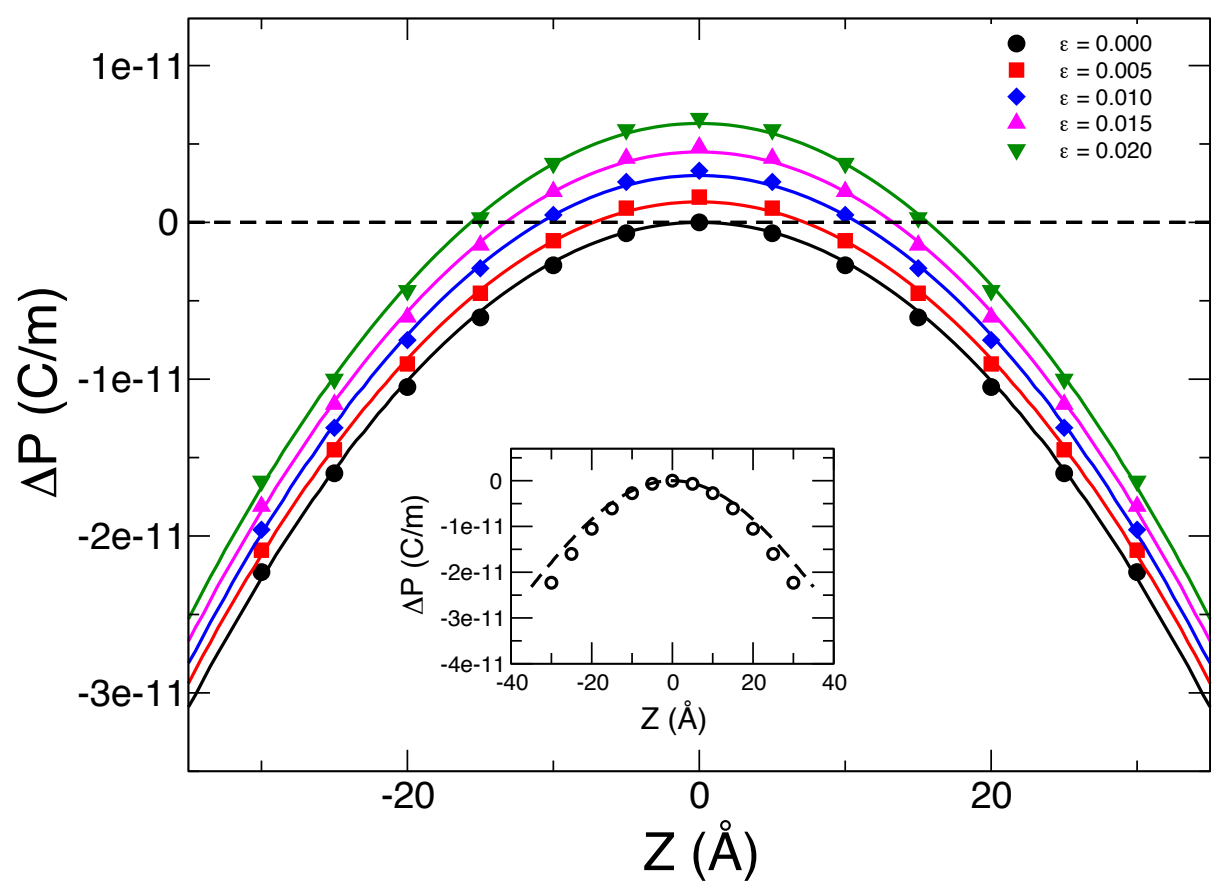

Figure 3. Electrical polarization as a function of the amplitude of the sinusoidal deformation for different values of the compressive strain $\epsilon$. The continuous lines are fits of the ab-initio data to a biquartic polynomial. The inset shows the good agreement between our calculated data (symbols) for $\epsilon=0$ and the phenomenological relation given in Naumov et al. [18] (dashed line), where the polarization is related to the amplitude of the sinusoidal deformation. 

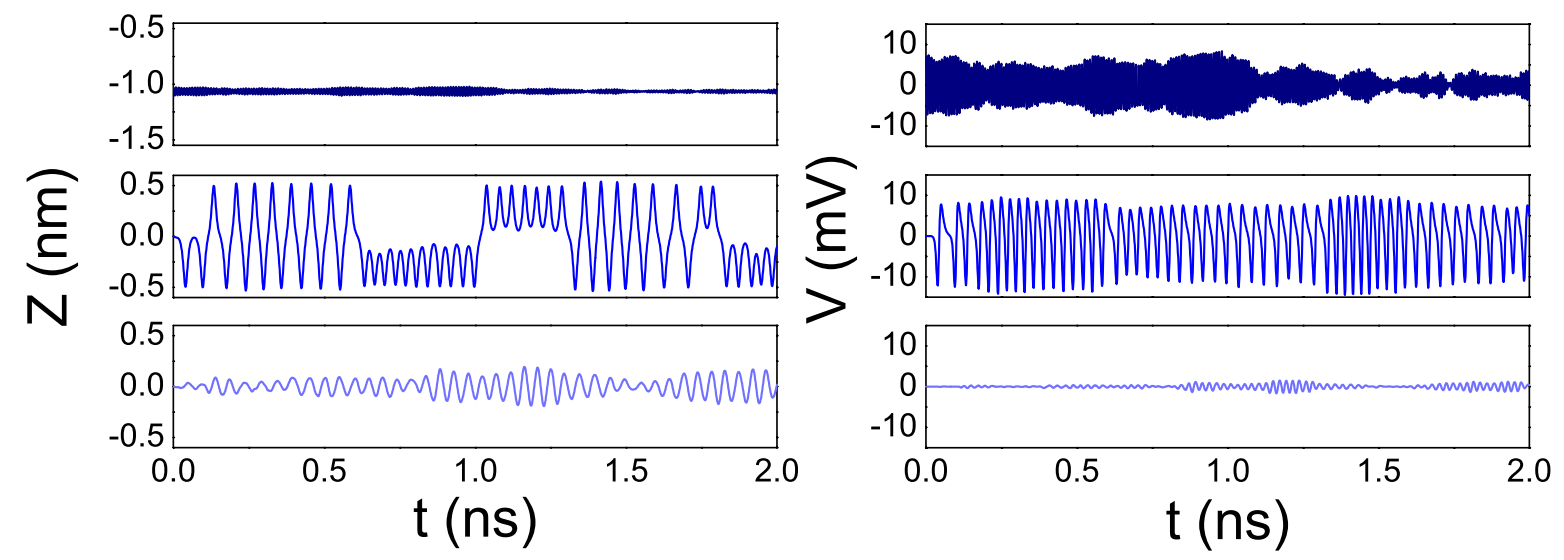

Figure 4. Time evolution of the generalized coordinate $z$ (left) and the generated voltage $V$ (right) in the three characteristic regimes: at no compression the single-well potential is quasi-harmonic (bottom); a moderate strain induces the bistable dynamics (middle); at large strains the dynamics is trapped in one of the minima of the doublewell potential (top). These simulations correspond to a $200 \AA \times 10 \AA$ h-BN monolayer. The strains applied in the middle and top panel are 0.3 and $1.0 \%$, respectively. 


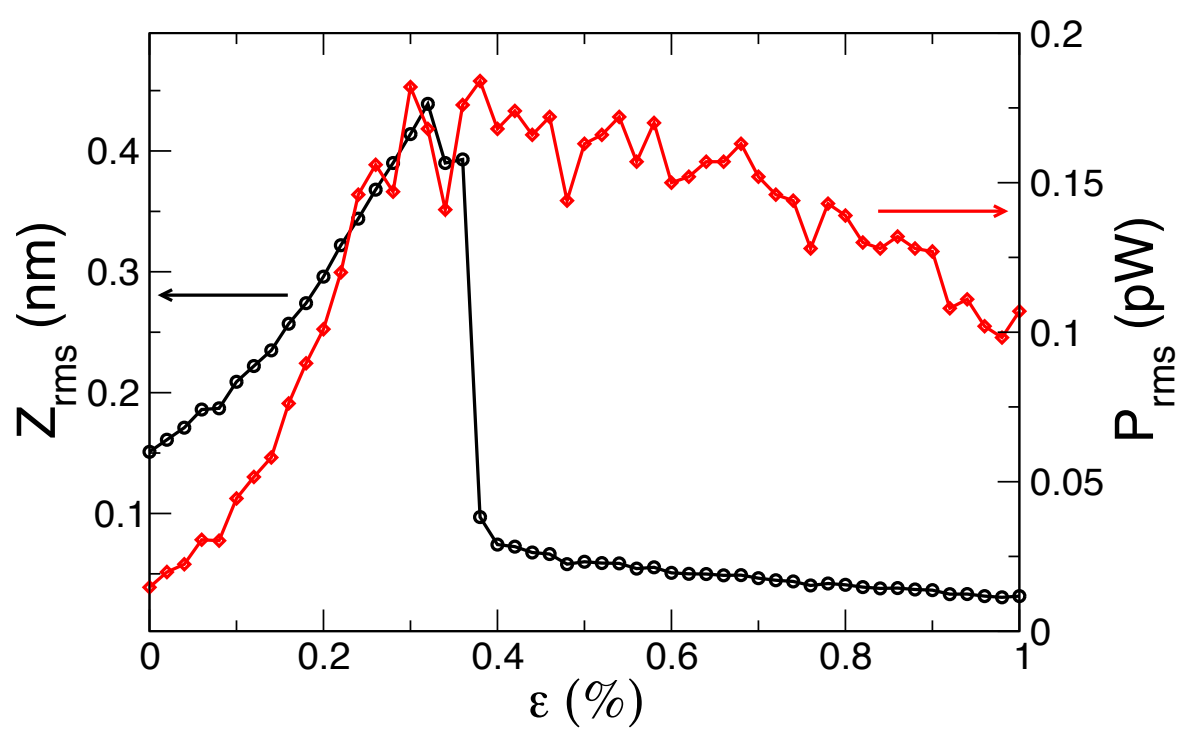

Figure 5. Root mean squares of $z$ and of the harvested electrical power as a function of the compressive strain $\epsilon$. The sudden drop of $z_{r m s}$ at large values of $\epsilon$ is balanced by the increase in $P_{x}^{2 D}$, thus the harvested power decreases gently after its maximum for the optimal compression. These simulations correspond to a $200 \AA \times 10 \AA \AA$ h-BN monolayer. 